\title{
A Road Surface Identification Method Improved Early Detection Performance Using Ultrasonic Sensors
}

\author{
Yudai Kubo a,, ${ }^{\text {, }}$ Hidemitsu Arimura ${ }^{a}$, Shenglin Mu ${ }^{b}$, Seiji Nishifujia ${ }^{a}$, Shota Nakashima ${ }^{a}$ \\ ${ }^{\mathrm{a}}$ Yamaguchi University, ${ }^{\mathrm{b}}$ Ehime University \\ a2-16-1, Tokiwadai, Ube, Yamaguchi, 755-8611, Japan \\ b3, Bunkyo-cho, Matsuyama, Ehime, 790-8577, Japan \\ *Corresponding Author: s-naka@yamaguchi-u.ac.jp
}

\begin{abstract}
Currently, the number of elderly people in the world is increasing. As a result, the number of accidents involving elderly people falling when movement is increasing. Development of mobility support systems is necessary for them to move safely. Therefore, the system was developed to help wheelchairs move by identifying the type of road surface in front of them. The system used ultrasonic sensors attached to the wheelchair to identify the road surface. Then, the method for identifying four types of road surfaces using Support Vector Machines (SVM) was proposed for the road surface identification method that constitutes the mobility support system. However, in the previous study, only the case where measured road surface didn't change was verified. This made it impossible to make early identification when the road surface changed during measurement. In this paper, the new road surface identification method using ultrasonic sensors is proposed. The proposed method makes it possible to identify the boundary of a road surface when it changes. In addition, the method improves the early detection performance. In order to verify the performance of early identification road boundary, two road surfaces with different roughness were measured in succession. As a result, the proposed method was able to identify at before entering the road boundary. This confirms the effectiveness of the road surface identification method that takes the time series into account for sample obtainment.
\end{abstract}

Keywords: ultrasonic sensor, road surface identification, support vector machine, mobility support system, involving falls.

\section{Introduction}

The number of elderly people in the world is increasing, and it is predicted that this trend will continue for several decades $^{(1)}$. Especially, the increase in the rate of elderly people in developed countries has become social problems. The problems include a declining workforce, a shortage of caregivers, and economic pressures due to the burden of medical costs. Accidents involving falls during mobility for the elderly are increasing as the population ages. Mobility aids such as wheelchairs, canes, and walkers are used to help elderly people move and prevent falls. These devices improve the safety of their daily lives ${ }^{(2)}$. On the other hand, elderly people acting alone are at high risk of falling, even when using mobility aids. To further improve the safety of elderly, it is necessary to develop mobility support systems. Currently, there are several mobility support systems that use sensors such as ultrasonic sensors, cameras, and lasers ${ }^{(3,4)}$. Ultrasonic sensors have the advantage among them that they are compact and have low computational cost compared to other sensors. The study has been conducted to detect steps and obstacles based on the distance information obtained by ultrasonic sensors ${ }^{(5-8)}$. However, the cause of falls is not only steps and obstacles, but also uneven surfaces such as grass and gravel. It is important to identify these surfaces in order to prevent falls. In the previous study, the road surface identification method was proposed using the feature that the road surface roughness depends on the ultrasonic reflection intensity ${ }^{(9)}$. Reflected waves is stronger on smooth surfaces and weaker on rough surfaces. This change is used to identify the road surface. Nevertheless, the previous study couldn't early identify when the road surface changed during the measurement. This is because the road surface can't be identified until the measurement of the sample size is completed. It was also necessary to measure additional 
sample sizes for the next road surface identification. In this study, in order to improve the performance of early road surface identification for the mobility support system, we build a classifier using SVM. In addition, samples are obtained by sliding one by one in the time series to verify the performance of early identification to the road surface change. This paper is organized as follows. The general introductions are in Section. Section II explain road surface identification method using the ultrasonic sensor. Section III, IV describes the experimental setup and the verification. Section V concludes the paper.

\section{A road surface identification using ultrasonic sensors}

\subsection{Measurement method}

This section describes the method of road surface identification using ultrasonic sensors. Fig. 1 shows a simplified diagram of the reflected wave measurement device. The device consists of two ultrasonic sensors (UT1612MPR/UR1612MPR), transmitters / receivers circuit, a microcomputer and a Bluetooth module. An ultrasonic sensor transmits by amplifying a $40 \mathrm{kHz}$ signal sent from the microcomputer with a transistor. The ultrasonic wave reflected from the target surface are then received an ultrasonic sensor, and the reflected wave is measured through amplification, half-wave rectification and envelope identification. Voltage-time integral is the integration of reflected wave in time ${ }^{(10)}$. Also, the reflected wave depends on three factors ${ }^{(11)}$. The first is the distance between the sensor and the road surface. Since ultrasonic waves are sound, it attenuates as it propagates through the medium. Furthermore, since ultrasonic propagates radially, the longer the distance, the weaker the reflected waves. The second is the incident angle of ultrasonic wave to the road surfaces. As this angle increases, the reflected wave sizes can be received decrease. Therefore, as this angle becomes smaller, the reflected waves become stronger. The third is the roughness

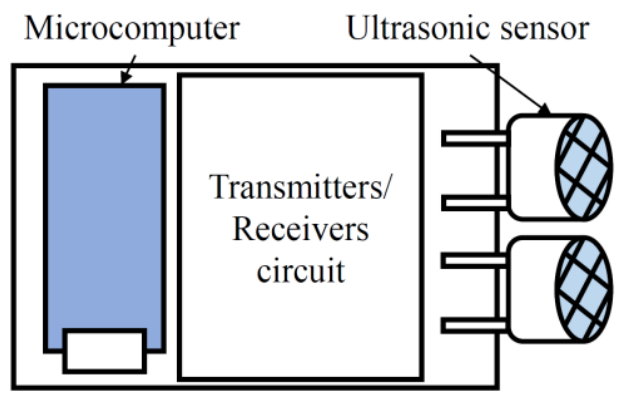

Fig. 1. Reflected wave measurement device. of the road surfaces. On rough surfaces, ultrasonic waves are scattered. As a result, the reflected wave received by the sensor becomes weaker. Also, on rough surfaces, the reflected waves change because the surface shape varies greatly from place to place. Fig. 2 shows measurement of reflected waves with different roughness and location. The black arrows show the strength of the reflected wave at each measurement location. The green arrows show the reflection of the ultrasonic waves. The reflected wave does not vary even if the measurement location is changed on smooth surfaces. On the other hand, the reflected waves vary from the different measurement location on rough surfaces. This is because the incident angles and reflection angles for the ultrasonic waves change irregularly due to the unevenness of the road surface. Thus, it is impossible to identify rough road surfaces by only measuring the reflected waves at the same measurement location. It is necessary to use the variation of the reflected wave as a parameter to identify the road surface roughness. The average and standard deviation of the voltage-time integrals were used to measure the variation of the road surface roughness. In this study, the voltage-time integrals are measured continuously while moving on the road surface. The measurement result is divided into multiple divisions by the number of samples. Then, the standardized average and standard deviation of each division are used as feature values to build SVM classifiers. Fig. 3 shows the method used to obtain the samples for SVM. The graph in Fig. 3 shows the obtained voltage-time integrals in chronological order. In the previous method, the voltagetime integrals are collected for the determined sample size. After that, the average and standard deviation were calculated, and this sample obtaining process was repeated. The time to obtain a sample is the time it takes for the voltage-time integrals to be measured for the sample size. In other words, it is impossible to early identify road surface change due to the longer time interval of the samples. Increasing the sample size also makes the correctness of the road surface identification higher ${ }^{(12)}$. In contrast, the sample size needs to be decreased to early identify the road surface. To solve the problem, the proposed method changes the way of obtaining samples. As in the previous method, the first sample is obtained by collecting the voltage-time integrals for the determined sample size. After that, the process of obtaining the next sample is repeated by sliding the obtainment range of the sample by one voltage-time integral. Since this method shortens the interval between samples, road surface changes can be early identified. If the sample size is reduced significantly, it isn't possible to identify the 


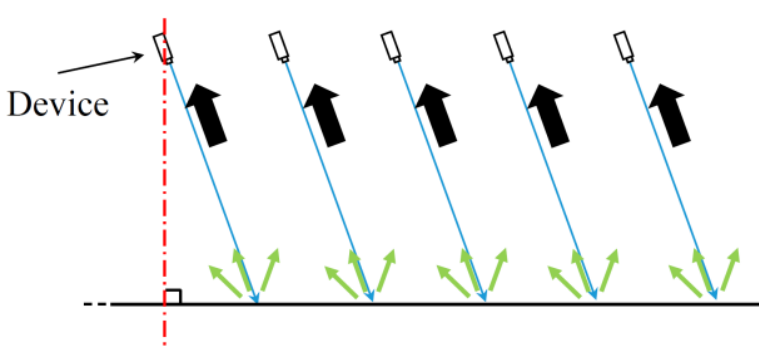

(a) Measurement on smooth surfaces.

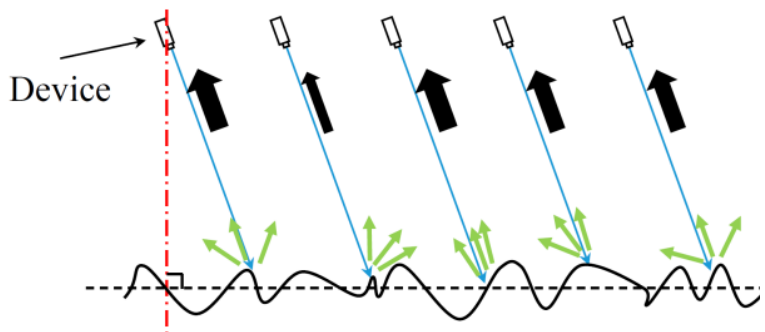

(b) Measurement on rough surfaces.

Fig. 2. Reflected wave on each measurement location.

road surface boundary accurately, because one data point has a greater influence on the average and standard deviation. In the experiment, the effectiveness of the sample size was also verified.

\section{$2.2 \quad$ SVM}

The measured voltage-time integrals are classified by SVM. It is a supervised learning algorithm designed to solve the binary classification problem ${ }^{(13)}$. It determines the hyperplane by maximizing the margin. The margin is the distance between the nearest data and the hyperplane. In the experiment, four different regularization parameters were tested with a soft margin SVM. It built a one-to-one classifier and performed multi-class classification. In addition, we used $\mathrm{C}=\{0.1,1,10,100\}$ as the regularization parameter. The data was divided into $70 \%$ of the training data and $30 \%$ of the test data. The percentage of accuracy score was verified for each parameter $C$. The correct answer is the label attached to the measurement.

\subsection{Evaluation value}

Vehicles don't stop immediately when the brakes are applied. There are situations where the brake is being operated but isn't working. The distance the vehicle drives in this situation is called the free running distance. The distance from when the brakes begin to act until the vehicle comes to stop is called the braking distance ${ }^{(14)}$. The sum of these two distances is called the stopping distance. $h \mathrm{~s}$ is the reaction speed. $v \mathrm{~km} / \mathrm{h}$ is the velocity of the vehicle. $\mu$ is the

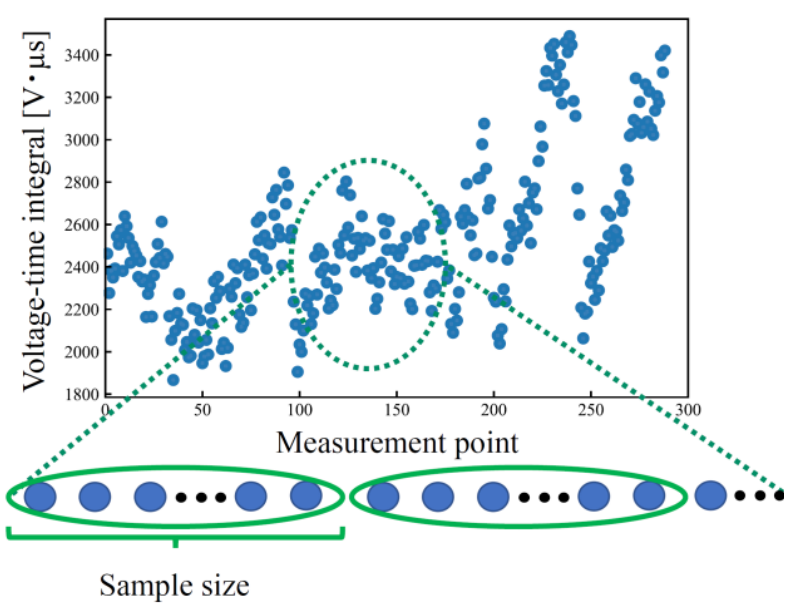

(a) Previous method

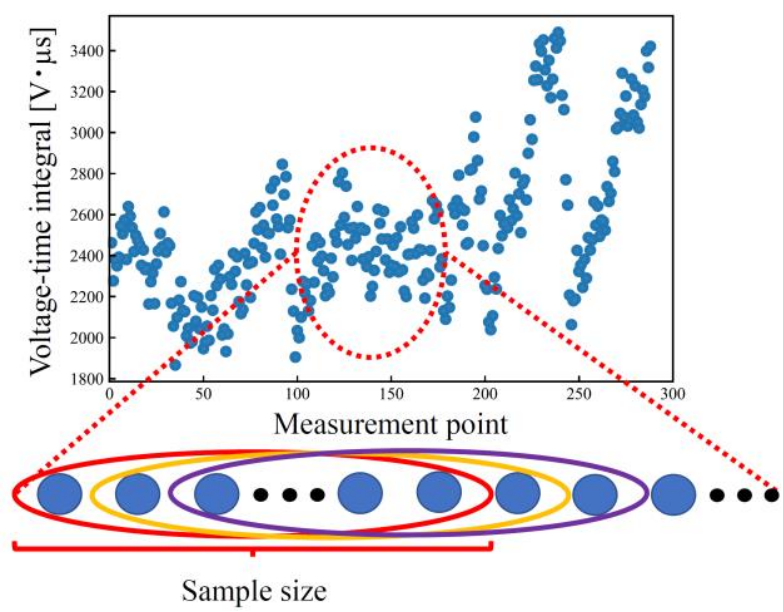

(b) Proposal method.

Fig. 3. Samples obtaining method.

coefficient of friction. It is between the tires and the road. $K$ $\mathrm{m}$ is the free running distance. $S \mathrm{~m}$ is the braking distance. $T$ $\mathrm{m}$ is the stopping distance. The free running, braking and stopping distances are described by equations (1), (2) and (3) respectively. The reaction rate and the friction coefficient were set to $h=1.0$ and $\mu=0.7$, respectively, since the experimental environment was a dry $\operatorname{road}^{(15)}$. The first moment when the road surface is identified as dangerous is explained. The distance between the front wheels and the road surface boundary is called the identification distance. tnd sec is the measured time of the first voltage-time integral to be identified as a dangerous road surface. $d c \mathrm{~m}$ is the distance from the start of measurement to the boundary distance. $v w \mathrm{~m} / \mathrm{s}$ is the average velocity of the wheelchair. $d i$ $\mathrm{m}$ is the identification distance. Equation (4) shows the identification distance.

$$
K=\frac{5 h v}{18}
$$




$$
\begin{aligned}
& S=\frac{v^{2}}{(254 \cdot \mu)} \\
& T=K+S \\
& d_{i}=d_{c}-v_{w} d_{n d}
\end{aligned}
$$

\section{Experiment}

\subsection{Setup}

To verify the effectiveness of the road surface identification method sampled by the proposed method by experiments. For this purpose, two experiments were conducted in sequence. The first was to verify the regularization parameters and sample size. The second is the early identification performance of road surface changes by the proposed method. The first experimental environment is described below. The measurement device was attached to a wheelchair, and the reflected waves were measured on the floor, mat, artificial turf, and gravel. This was done in order to keep the distance constant and limit the factor of change in the reflected waves to the road surface roughness. The device was attached to the right armrest extension of the wheelchair. The incident angle of ultrasonic was set at $20 \mathrm{deg}$.

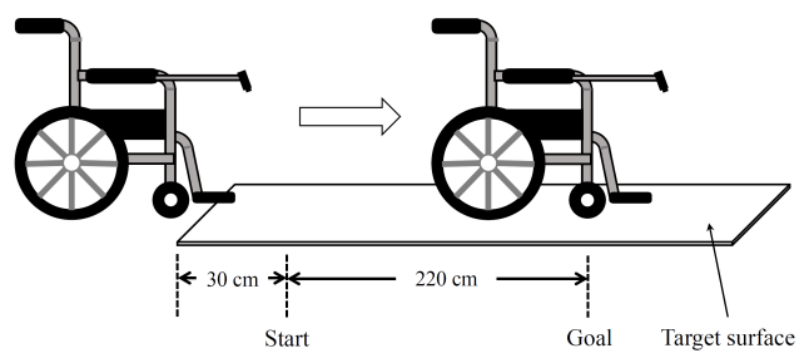

(a) Verification experiments for

regularization parameters and sample size

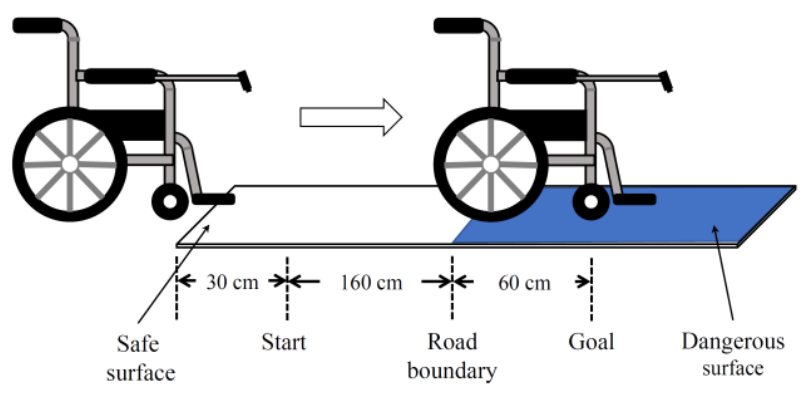

(b) Verification experiment for early identification of road surface changes

Fig. 4. Experimental environment.
The distance between the sensors and the road surface was $60 \mathrm{~cm}$. Fig. 4 shows a simplified representation of the experimental environment. In Fig. 4, there were four types of surfaces to be measured: floor, mat, artificial turf, and gravel. The measurement procedure of the reflected waves is described below. The wheelchair equipped with the measurement device is placed $30 \mathrm{~cm}$ in front of the start position shown in Fig. 4. The wheelchair was pushed in a straight line. Measurements were started at the starting point and continued until the front wheels of the wheelchair reached the goal. This flow was taken as one set, and 30 sets of measurements were taken for each road surface. The labeled voltage-time integral for each road surface were obtained. All measurement sets for each label were ordered and sampled by sliding them one by one with the determined sample size. The sample size was set to $\mathrm{N}=\{100,200,300$, $400\}$. Fig. 4(b) shows the environment of the second experiment The equipment and mounting of the measuring device were the same as in the first experiment. The target surfaces for measurement in Fig. 4 are four types of road surfaces that change from safe to dangerous. The safe surfaces are floors and mats, and the dangerous surfaces are artificial turf and gravel. These two types of road surfaces were set up so as to keep the measurement range of the device the same. The number of sets was changed to 20 and the method of obtaining the samples was the same as in the first experiment. The identification distance was calculated from equation 4 . The identification distance was compared to the stopping distance.

\subsection{Result and discussion}

Fig. 5 shows a classifier built using SVM with the average and standard deviation of voltage-time integral as the features. The $\mathrm{X}$-axis is the average, and the $\mathrm{Y}$-axis is the standard deviation. Tables 1 and 2 show the percentage of correct answers to the training and test data when the sample size and regularization parameters are changed. From the experimental results, the sample size and regularization parameters of the classifier are considered. The classifier built with the proposed method has accuracy rate of more than $90 \%$ for both training and test data. The correct answer rate exceeded $95 \%$ when the sample size was larger than 200 . Therefore, the sample size used in the early detection of road surface changes was determined to be 200 . This result is true for all the sample sizes considered in this study. The accuracy scores didn't change by more than $1 \%$ when the regularization parameters were changed. Thus, the five 
regularization parameters tested in this experiment are considered to have little effect on the classifier. The regularization parameter used in the early detection of road surface changes was determined to be $C=1$. Fig. 6 and Fig. 7 show the transition of the road surface on the classifier when the road surface changes from floor to dangerous road surface and from mat to dangerous road surface, respectively. Table 2 shows the identification distance, the stopping distance and boundary distance for each road surface change. Fig. 6 and Fig. 7 show that the transitions at each road surface change are finely identified. Table 3 also shows that it is possible to identify the road surface before the front wheels

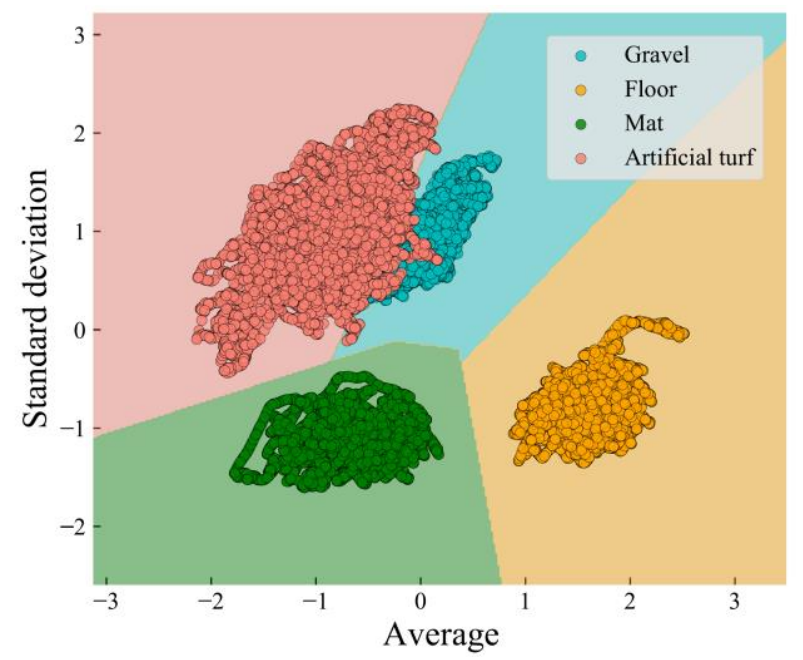

Fig. 5. Classifier.

Table. 1. Percentage of correct answers to the training data

\begin{tabular}{|c|c|c|c|c|c|}
\hline \multirow{2}{*}{ Sample size } & \multicolumn{5}{|c|}{$C$} \\
\cline { 2 - 6 } & 0.01 & 0.1 & 1 & 10 & 100 \\
\hline 100 & 94.8 & 94.8 & 94.8 & 94.8 & 94.8 \\
\hline 200 & 97.8 & 98.1 & 98.1 & 98.1 & 98.1 \\
\hline 300 & 99.4 & 99.6 & 99.6 & 99.6 & 99.6 \\
\hline 400 & 99.9 & 99.9 & 99.9 & 99.9 & 100 \\
\hline
\end{tabular}

Table. 2. Percentage of correct answers to the test data

\begin{tabular}{|c|c|c|c|c|c|}
\hline \multirow{2}{*}{ Sample size } & \multicolumn{5}{|c|}{$C$} \\
\cline { 2 - 6 } & 0.01 & 0.1 & 1 & 10 & 100 \\
\hline 100 & 94.7 & 94.7 & 94.7 & 94.8 & 94.8 \\
\hline 200 & 97.9 & 98.1 & 98.1 & 98.1 & 98.1 \\
\hline 300 & 99.2 & 99.4 & 99.5 & 99.5 & 99.5 \\
\hline 400 & 99.9 & 99.9 & 99.9 & 99.9 & 99.9 \\
\hline
\end{tabular}

of the wheelchair enter the dangerous surface. On the other hand, when the stopping distance was taken into account, the wheelchair couldn't identify before the stopping distance when the safe surface was the floor. The reason for this could be the difference in the voltage-time integral and the shaking of the wheelchair. The differences in the voltage-time integrals are mentioned. The average of the voltage-time integral of the floor is much larger than that of the dangerous surface. In addition, the standard deviation is much smaller for the floor than for the dangerous surface. For these reasons, the distributions of the floor and the dangerous road surface are opposite to each other on the classifier. This caused a delay in identifying road surface changes. The shaking of the wheelchair is mentioned. In the first experiment, the wheelchair was actually driven over all road surfaces. The wheelchair was shaking while measuring the voltage-time integral on the gravel surface. On the other hand, in the

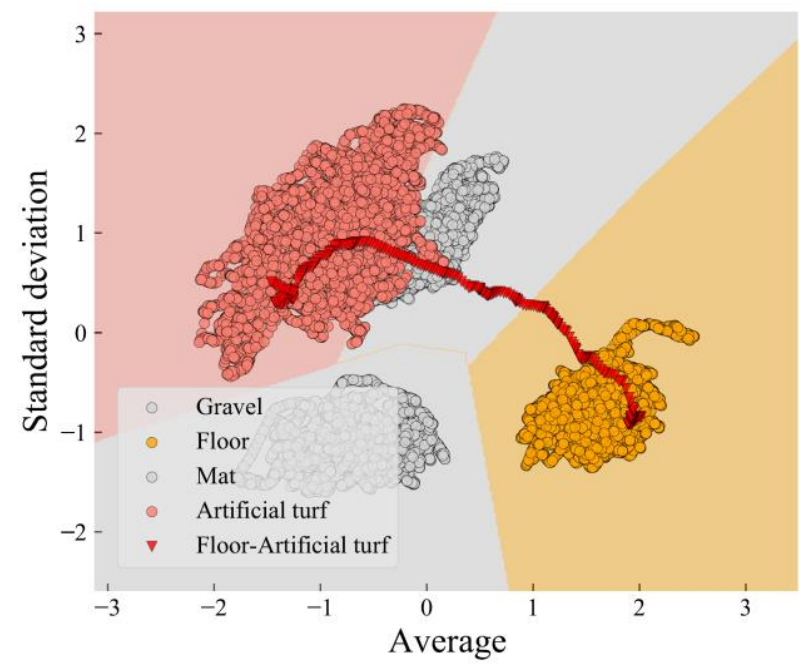

(a) Floor to Artificial turf

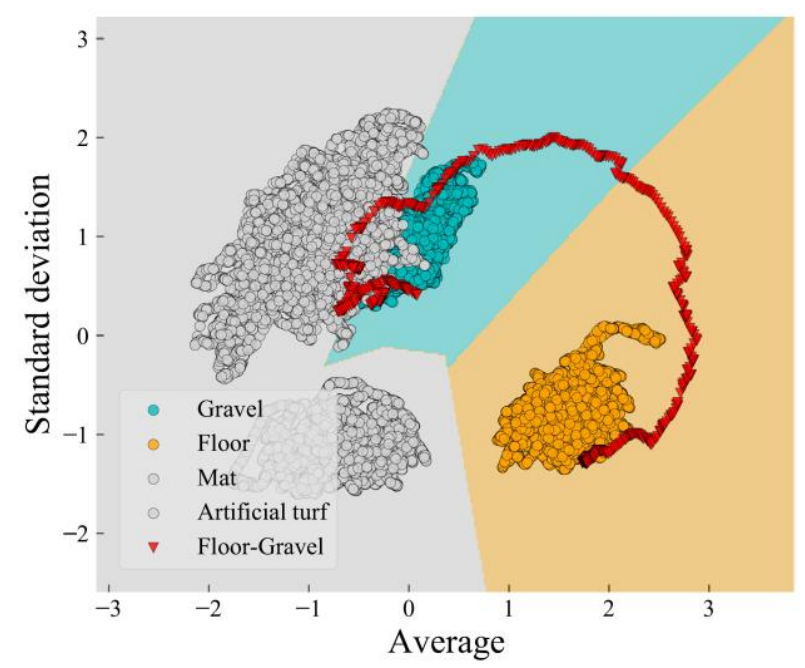

(b) Floor to Gravel 


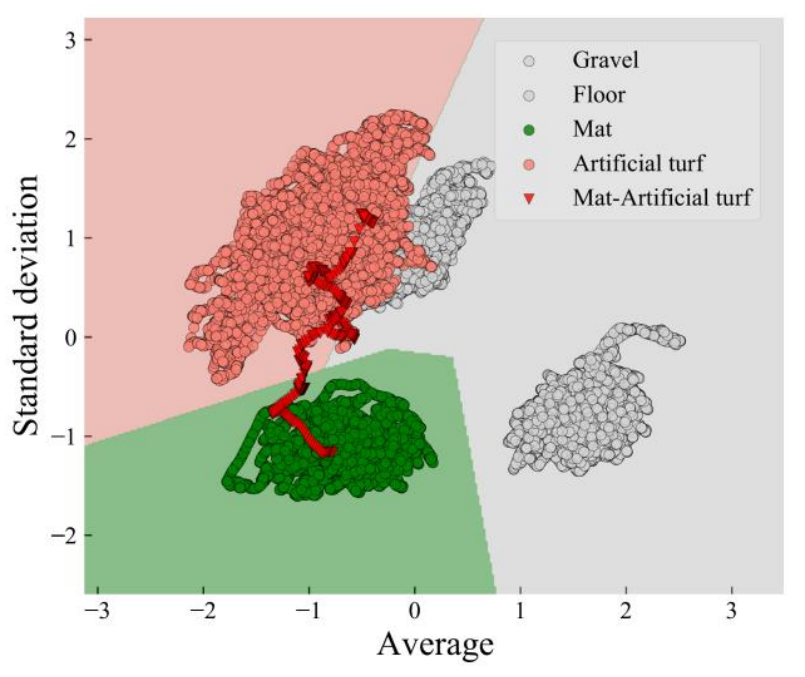

(a) Mat to Artificial turf

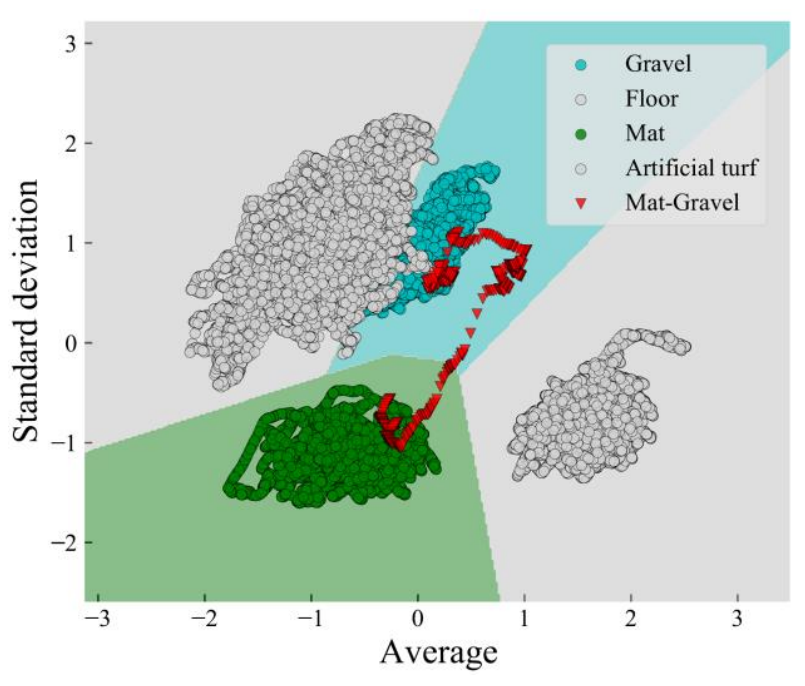

(b) Mat to Gravel

Fig. 7. Change from mat to dangerous surface.

Table. 3. Relationship between identification and stopping distances

\begin{tabular}{|c|c|c|c|}
\hline & $\begin{array}{c}\text { Wheelchair } \\
\text { velocity } \\
{[\mathrm{cm} / \mathrm{s}]}\end{array}$ & $\begin{array}{c}\text { Identification } \\
\text { distance } \\
{[\mathrm{cm}]}\end{array}$ & $\begin{array}{c}\text { Stopping } \\
\text { distance } \\
{[\mathrm{cm}]}\end{array}$ \\
\hline $\begin{array}{c}\text { Floor to } \\
\text { Artificial turf }\end{array}$ & 27.2 & 19.8 & 27.8 \\
\hline $\begin{array}{c}\text { Floor to } \\
\text { Gravel }\end{array}$ & 19.3 & 12.7 & 19.6 \\
\hline $\begin{array}{c}\text { Mat to } \\
\text { Artificial turf }\end{array}$ & 27.5 & 45.3 & 28.0 \\
\hline $\begin{array}{c}\text { Mat to } \\
\text { Gravel }\end{array}$ & 18.7 & 51.9 & 19.0 \\
\hline
\end{tabular}

second experiment, the measurement distance was determined by considering the measurement range. Therefore, most of the measurements for the dangerous road surface were taken from the safe road surface. This resulted in less swaying during the measurement. The average of the gravel differed greatly between the two experiments. These results indicate that the averages of the floor and gravel were close to each other, and it took time to identify them.

\section{Conclusions}

In this paper, the road surface identification method using ultrasonic sensors was proposed. In this method, samples are obtained by sliding them one by one in a time series, which enables early detection of road surface changes. The accuracy score of the proposed method was verified by experiments, and the results showed that the accuracy was high and the number of samples that could be obtained increased. In addition, the method was also able to early identify the road surface changes before entering the dangerous road surface. The method can be applied to mobility support systems for elderly people, visually impaired people, and automobiles.

\section{Acknowledgment}

This work was supported by JSPS KAKENHI Grant Number JP21K12797.

\section{References}

(1) United Nations, "World Population Prospects 2019: Highlights" Department of Economic and Social Affairs, 2019

(2) V. Nandapalan, C.A. Smith, A.S. Jones and T.H. Lesser : "Objective measurement of the benefit of walking sticks in peripheral vestibular balance disorders, using the Sway Weigh balance platform", J Laryngol Otol, Vol.109, No.9, pp. 836-840, 1995

(3) S. Pundlik, V. Baliutaviciute, M. Moharrer, A.R. Bowers and G. Luo : "Data Acquisition, Processing, and Reduction for Home-Use Trial of a Wearable Video Camera-Based Mobility Aid", Translational Vision Science \& Technology, Vol. 9, No. 7, 2020

(4) G. Yamamoto, A. Chen, P. Pulli, J. Hyry, M.Z. Asghar, Y. Uranishi and H. Kato : "A laser projection-based teleguidance system embedded on a mobility aid", 7th International Symposium on Medical Information and Communication Technology, 2013 
(5) D.S. Vidhya, D. Perlin Rebelo, C. J. D’Silva, L.W. Fernandes and C. J. Costa : "Obstacle detection using ultrasonic sensors", International Journal for Innovative Research in Science \& Technology, Vol. 2, No. 11, 2016

(6) N. Gageik, T. Müller and S. Montenegro : "Obstacle detection and collision avoidance using ultrasonic distance sensors for an autonomous quadrocopter", Proc. UAVveek Workshop Contrib., 2012

(7) C. N. Yalung and C.S. Mathew Adolfo : "Analysis of Obstacle Detection Using Ultrasonic Sensor", International Research Journal and Engineering Technology, pp. 1015-1019, 2017.

(8) H. Dobashi, T. Tajima, T. Abe and H. Kimura : "Fall Detection System for Bather Using Ultrasound Sensors", Proceedings of 9th Asia Pasific Industrial Engineering \& Management Systems Conference, Nusa Dua, Bali, Indonesia, 2008.

(9) M. Yamamoto, S. Nakashima, K. Haruyama, and S. Mu : "Improvement of Road Surface Discrimination Performance of Movement Support System Using Ultrasonic Sensors", Int. Conf. on Applied Comp. and Information Technology, No. 33, pp. 1-6, 2019

(10) H. Arimura, M Yamamoto, S. Mu and S. Nakashima : "A Road Surface Distinction Method Based on Integral of Reflected Wave Using Ultrasonic Sensors", International Symposium on Computer, Consumer and Control, pp. 541-544, 2020

(11) S. Nakashima, S. Aramaki, Y. Kitazono, S. Mu, K. Tanaka and S. Serikawa : "Application of Ultrasonic Sensors in Road Surface Condition Distinction Methods", 2016

(12) S. Nakashima, H. Arimura, M. Yamamoto, S. Mu and H. $\mathrm{Lu}$ : "Improving the Accuracy of Road Surface Distinction Based on Reflection Intensity Variations Using Ultrasonic Sensor”, IEEE SENSORS JOURNAL, pp. 1-7, 2020

(13) J. Shawe-Taylor and N. Cristianni : "An introduction to support vector machines and other kernel-based learning methods", Cambridge University, 2000

(14)R. Sun, X. Zhuang, C. Wu, G. Zhao and K. Zhang : "The estimation of vehicle speed and stopping distance by pedestrians crossing streets in a naturalistic traffic environment", Transp. Res. Part F, Vol.30, pp. 97-106, 2015

(15) American Association of State Highway and Transportation Officials : "A Policy on Geometric Design of Highways and Streets", AASHTO, 2004 